\title{
Applications of the indole-alkaloid gramine modulate the assembly of individual members of the barley rhizosphere microbiota
}

\author{
Mauro Maver ${ }^{1,2,3}$, Carmen Escudero-Martinez ${ }^{1}$, James Abbott ${ }^{4}$, Jenny Morris ${ }^{5}$, Pete Hedley ${ }^{5}$, Tanja $^{\text {Mimmo }}{ }^{2,3}$, \\ Davide Bulgarelli ${ }^{\text {Corresp. } 1}$ \\ 1 Plant Sciences, School of Life Sciences, University of Dundee, Dundee, United Kingdom \\ 2 Faculty of Science and Technology, Free University of Bozen-Bolzano, Bolzano, Italy \\ ${ }^{3}$ Competence Centre for Plant Health, Free University of Bozen-Bolzano, Bolzano, Italy \\ 4 Data Analysis Group, School of Life Sciences, University of Dundee, Dundee, United Kingdom \\ ${ }^{5}$ Cell and Molecular Sciences, The James Hutton Institute, Dundee, United Kingdom \\ Corresponding Author: Davide Bulgarelli \\ Email address: d.bulgarelli@dundee.ac.uk
}

Microbial communities proliferating at the root-soil interface, collectively referred to as the rhizosphere microbiota, represent an untapped beneficial resource for plant growth, development and health. Integral to a rational manipulation of the microbiota for sustainable agriculture is the identification of the molecular determinants of these communities. In plants, biosynthesis of allelochemicals is centre stage in defining interorganismal relationships in the environment. Intriguingly, this process has been moulded by domestication and breeding selection. The indole-alkaloid gramine, whose occurrence in barley (Hordeum vulgare L.) is widespread among wild genotypes but has been counter selected in several modern varieties, is a paradigmatic example of this phenomenon. This prompted us to investigate how exogenous applications of gramine impacted on the rhizosphere microbiota of two, gramine-free, elite barley varieties grown in a reference agricultural soil. High throughput 16S rRNA gene amplicon sequencing revealed that applications of gramine interfere with the proliferation of a subset of soil microbes with a relatively broad phylogenetic assignment. Strikingly, growth of these bacteria appeared to be rescued by barley plants in a genotype- and dosage-independent manner. In parallel, we discovered that host recruitment cues can interfere with the impact of gramine application in a host genotype-dependent manner. Interestingly, this latter effect displayed a bias for members of the phyla Proteobacteria. These initial observations indicate that gramine can act as a determinant of the prokaryotic communities inhabiting the root-soil interface. 
1 Applications of the indole-alkaloid gramine modulate the assembly of

2 individual members of the barley rhizosphere microbiota

3

4 Mauro Maver ${ }^{1,2,3}$, Carmen Escudero-Martinez ${ }^{2}$, James Abbott ${ }^{4}$, Jenny Morris ${ }^{5}$, Pete E. Hedley ${ }^{5}$,

5 Tanja Mimmo ${ }^{1,3^{*}}$ and Davide Bulgarelli ${ }^{*}$

$6 \quad{ }^{1}$ Faculty of Science and Technology, Free University of Bozen-Bolzano, Bolzano, Italy

$7 \quad 2$ Plant Sciences, School of Life Sciences, University of Dundee, Dundee, United Kingdom

$8{ }^{3}$ Competence Centre for Plant Health, Free University of Bozen-Bolzano, Bolzano, Italy

$9 \quad{ }^{4}$ Data Analysis Group, School of Life Sciences, University of Dundee, Dundee, United

10 Kingdom

$11{ }^{5}$ Cell and Molecular Sciences, The James Hutton Institute, Dundee, United Kingdom

12

$13 *$ Corresponding Authors:

14

15 Tanja Mimmo

16 Faculty of Science and Technology, Free University of Bozen-Bolzano, Bolzano, Italy

17 Email address: tmimmo@unibz.it

18

19 Davide Bulgarelli

20 Plant Sciences, School of Life Sciences, University of Dundee, Dundee, United Kingdom

21 Email address: d.bulgarelli@dundee.ac.uk 


\section{Abstract}

25

26 Microbial communities proliferating at the root-soil interface, collectively referred to as the 27 rhizosphere microbiota, represent an untapped beneficial resource for plant growth, development 28 and health. Integral to a rational manipulation of the microbiota for sustainable agriculture is the 29 identification of the molecular determinants of these communities. In plants, biosynthesis of 30 allelochemicals is centre stage in defining inter-organismal relationships in the environment. 31 Intriguingly, this process has been moulded by domestication and breeding selection. The indole32 alkaloid gramine, whose occurrence in barley (Hordeum vulgare L.) is widespread among wild 33 genotypes but has been counter selected in several modern varieties, is a paradigmatic example 34 of this phenomenon. This prompted us to investigate how exogenous applications of gramine 35 impacted on the rhizosphere microbiota of two, gramine-free, elite barley varieties grown in a 36 reference agricultural soil. High throughput 16S rRNA gene amplicon sequencing revealed that 37 applications of gramine interfere with the proliferation of a subset of soil microbes with a 38 relatively broad phylogenetic assignment. Strikingly, growth of these bacteria appeared to be 39 rescued by barley plants in a genotype- and dosage-independent manner. In parallel, we 40 discovered that host recruitment cues can interfere with the impact of gramine application in a 41 host genotype-dependent manner. Interestingly, this latter effect displayed a bias for members of 42 the phyla Proteobacteria. These initial observations indicate that gramine can act as a 43 determinant of the prokaryotic communities inhabiting the root-soil interface. 


\section{Introduction}

45

46 The interface between roots and soil hosts distinct microbial communities, collectively referred

47 to as rhizosphere microbiota (Turner, James \& Poole, 2013). These plant-microbial assemblages

48 define a continuum of parasitic, commensal, and mutualistic interactions (Schlaeppi \&

49 Bulgarelli, 2015). For example, so-called plant growth-promoting rhizobacteria can enhance

50 plant mineral uptake and protect their hosts from pathogens (Lugtenberg \& Kamilova, 2009).

51 Studies conducted with multiple plant species support the notion that members of the rhizosphere

52 microbiota are not passively assembled from the surrounding soil biota, rather a multi-step

53 selection process differentiate plant-associated communities from those identified in unplanted

54 soils (Bulgarelli et al., 2013; Edwards et al., 2015). This selection process operates at multiple

55 taxonomic and functional ranks, with the enrichment of members of the phyla Actinobacteria,

56 Bacteroidetes, Proteobacteria and Firmicutes representing the distinct signature of plant-

57 associated communities (Bulgarelli et al., 2013; Alegria Terrazas et al., 2016). The host genome

58 represents one of the determinants in the selection process defining the microbiota thriving at the

59 root-soil interface (Schlaeppi \& Bulgarelli, 2015; Hacquard et al., 2015; Alegria Terrazas et al.,

60 2016). This selection is exerted through a number of plant traits, including root system

61 architecture (Robertson-Albertyn et al., 2017; Pérez-Jaramillo et al., 2017) and the plant immune

62 system (Lebeis et al., 2015). Another key element of the host-mediated molecular mechanisms

63 shaping the rhizosphere microbiota is the root exudation of metabolites capable of modulating

64 the interactions among plants, microbes and the surrounding environment (Dakora \& Phillips,

65 2002; Jones, Nguyen \& Finlay, 2009; Pascale et al., 2020). Consistently, an increasing number of 
66 plant primary (Canarini et al., 2019), as well as secondary (Rolfe, Griffiths \& Ton, 2019)

67 metabolites have recently been implicated in shaping the plant microbiota.

68 Modern crops are the result of an on-going selection process, initiated with domestication and 69 continued with breeding selection, which progressively differentiated cultivated varieties from

70 their wild ancestors (Purugganan \& Fuller, 2009). Interestingly, these selection processes

71 impacted on both plant's ability to assemble a rhizosphere microbiota (Pérez-Jaramillo, Mendes

$72 \&$ Raaijmakers, 2016) and its capacity of secreting metabolites at the root-soil interface (Preece $73 \&$ Peñuelas, 2020).

74 As wild ancestors of modern cultivated varieties may hold the capacity to adapt to marginal soil 75 conditions, there is a growing interest in discerning the molecular mechanisms underpinning 76 microbiota recruitment in crop wild relatives and their contribution to plant's adaptation to the 77 environment (Escudero-Martinez \& Bulgarelli, 2019). This is particularly attractive for crops 78 like barley (Hordeum vulgare), the fourth most cultivated cereal worldwide, for which modern 79 and wild genotypes are readily available for experimentation (Bulgarelli et al., 2015; Alegria 80 Terrazas et al., 2020).

81 The genus Hordeum has evolved two main indole alkaloids with allelopathic and defensive 82 functions, the benzoxazinoid DIBOA and gramine, whose biosynthesis appear mutually 83 exclusive within barley lineages (Grün, Frey \& Gierl, 2005). In particular, gramine is the main 84 allelochemical of the lineage $H$. vulgare which has historically been implicated in defensive 85 responses against insects (Corcuera, 1993; Cai et al., 2009; Sun et al., 2013), as well as foliar 86 pathogens (Sepulveda \& Corcuera, 1990; Matsuo et al., 2001) although the genetic basis of this 87 trait appears complex (Åhman, Tuvesson \& Johansson, 2000; Macaulay, Ramsay \& Åhman, 88 2020). Intriguingly, crop selection left a footprint on the biosynthesis of this secondary 
89 metabolite: modern cultivated, so called 'elite', varieties (H. vulgare subp. vulgare) often fail to

90 accumulate gramine to levels identified in their wild relatives (H. vulgare subp. spontaneum)

91 (Matsuo et al., 2001; Maver et al., 2020). Of note, this apparent counter-selection for gramine

92 within domesticated material has been exerted on at least two distinct biosynthetic genes in

93 barley (Larsson et al., 2006).

94 To gain novel insights into the ecological significance of gramine for plant-microbiota

95 interactions, we hypothesized that the release of this secondary metabolite act as a recruitment

96 cue for the barley microbiota. Despite F1 hybrids between wild and domesticated genotypes

97 producing gramine do exist (Moharramipour et al., 1999), no barley isogenic lines for the

98 biosynthesis of this secondary metabolites are currently accessible to experimentation. This

99 makes it difficult to discriminate between gramine and other genetic factors putatively impacting

100 on microbiota composition. We therefore decided to test our hypothesis, by exposing two 'elite',

101 gramine-free, barley genotypes, the cultivars Morex and Barke (Larsson et al., 2006), to

102 exogenous applications of gramine and we assessed the impact of these treatments on the

103 taxonomic composition of the prokaryotic microbiota thriving at the root-soil interface using a

104 cultivation independent approach. 
106 Materials \& Methods

107 Soil substrate

108 The experiments were carried out in "Quarryfield” soil, an unfertilized Scottish agricultural soil

109 collected at the site of The James Hutton Institute, Invergowrie, Scotland (UK) (56 $27^{\prime} 5^{\prime \prime} \mathrm{N}$

$\left.1103^{\circ} 4^{\prime} 29^{\prime \prime}\right)$. This soil was used previously to grow barley and left unplanted at least 4 years

111 before being used for the experiments. Physical and chemical characterization: Silt: 39.35\%;

112 Clay: 11.08\%; Sand: 49.58\%; pH in water: 5.8; C.E.C.: 15.05 [meq/100g]; Org. Matter DUMAS:

$1135.95 \%$.

114

115 Gramine adsorption

116 Five grams of Quarryfield soil were mixed with $10 \mathrm{~mL}$ of $10 \mathrm{mmol} \mathrm{L}^{-1} \mathrm{CaCl}_{2}$ solution containing

117 gramine at the following concentrations: $0,1.5,5,10,15,20,30,60,100,150,200,250,300$,

$118400,600,800,1000 \mathrm{mg} \mathrm{L}^{-1}$. Five replicates were prepared for each concentration. Soil

119 suspensions were shaken for $24 \mathrm{~h}$ at room temperature. Then, suspensions were centrifuged for 5

$120 \mathrm{~min}$ at $5000 \mathrm{~g}$, the supernatant was collected, filtered $(0.45 \mu \mathrm{m}$, Phenomenex $)$ and analysed by

121 liquid chromatography (HPLC) using the method reported in (Maver et al., 2020). Pure reagent

122 grade gramine was used to prepare two stock solutions in water of $0.5 \mathrm{~mol} \mathrm{~L}^{-1}$ and $10 \mathrm{mmol} \mathrm{L}^{-1}$.

123 Adsorption solutions have been prepared by dilution from the stock solution with milliQ water.

124 The adsorption rate of gramine in soil was obtained by difference between the initial and final

125 concentration measured in the supernatant by HPLC.

126 Adsorption isotherms of gramine were fitted applying several nonlinear models: the two-

127 parameter Langmuir and Freundlich isotherms and the three-parameter Sigmoidal Langmuir,

128 Redlich-Peterson and Sips isotherms (Limousin et al., 2007; Foo \& Hameed, 2010). 
130

131 Seeds of elite barley genotypes, two-row malting Barke and six-row malting Morex were

132 selected for this experiment. Both are well represented in barley studies: Barke as a parental

133 donor in the development of a nested associated mapping (NAM) population (Maurer et al.,

134 2015), and Morex for being the first sequenced barley genotype (The International Barley

135 Genome Sequencing Consortium, 2012).

136

137

138

139

140

141

142

143

144

145

146

147

148

149

150

151

152

\section{Growth conditions}

Barley seeds were cleaned using deionized water and gently shaken for $1 \mathrm{~min}$. After that water was discarded and the whole process repeated 3 times. For seed germination, seeds were placed on petri dishes containing a semi-solid $0.5 \%$ agar solution. After a week, seedlings displaying a comparable development were individually transferred into $12-\mathrm{cm}$ pots containing approximately 500 g of Quarryfield agricultural soil (Robertson-Albertyn et al., 2017; Alegria Terrazas et al., 2020) previously sieved to remove stones and large debris. Plants were grown in a randomized design in a glasshouse at $18 / 14^{\circ} \mathrm{C}$ (day/night) temperature regime with $16 \mathrm{~h}$ daylight that was supplemented with artificial lighting to maintain a minimum light intensity of $200 \mu$ mol quanta $\mathrm{m}^{-2} \mathrm{~s}^{-1}$. The stock solution of gramine was prepared by adding pure reagent grade gramine

47 (Sigma-Aldrich, $>99 \%$ ), in water, sonicated for $20 \mathrm{~min}$ and then stored at $4{ }^{\circ} \mathrm{C}$. After 4 days of growing in soil, two different final gramine concentrations, $24 \mu \mathrm{mol} \mathrm{L}-1$ and $46 \mu \mathrm{mol} \mathrm{L}-1$, were added directly on the soil of selected pots. Mock controls (i.e., gramine $0 \mu \mathrm{mol} \mathrm{L}{ }^{-1}$ ) were supplemented with sterilised water. Additional watering was performed every 2 days with the application of $50 \mathrm{~mL}$ of deionized water to each pot. For each gramine treatment (i.e., 0, 24 and $46 \mu \mathrm{mol} \mathrm{L}{ }^{-1}$ ) we used five replicates (i.e., five individual pots) per barley genotype and 
153 unplanted pots containing the same soil substrates used as 'bulk' soil controls (i.e., 45 pots in

154 total). Individual replicated pots were maintained in the glasshouse for 4 weeks post-

155 transplantation, when the tested genotypes reached early stem elongation, corresponding to

156 Zadoks stages 30-35 (Tottman, Makepeace \& Broad, 1979).

157

158

Rhizosphere fractionation and sampling of soil-grown barley plants and bulk soil

159 The preparation of material for amplicon sequencing was performed following established

160 protocols (Robertson-Albertyn et al., 2017; Alegria Terrazas et al., 2020). Briefly, Four-week-

161 old barley plants were carefully removed from the soil, and the shoot and root separated. The

162 shoot was dried at $70^{\circ} \mathrm{C}$ for $48 \mathrm{~h}$ and the dry weight collected. The roots were gently shaken to

163 remove loosely bound soil particles, and the resulting root system and tightly adhered soil,

164 operationally defined as rhizosphere, was further sectioned to retain the uppermost $6 \mathrm{~cm}$ of the

165 seminal root system of each sample. This root material was transferred in a sterile $50 \mathrm{~mL}$ falcon

166 tube containing $15 \mathrm{~mL}$ of phosphate buffered saline solution (PBS). Samples were then vortexed

167 for $30 \mathrm{~s}$, the soil sedimented for 2-3 mins, and transferred the roots in a new $50 \mathrm{~mL}$ falcon tube

168 with $15 \mathrm{~mL}$ PBS, in which the samples were vortexed again for $30 \mathrm{~s}$ to separate the remaining

169 rhizosphere soil from roots. The roots were discarded, the two falcon tubes were combined in

170 one single falcon tube, now containing the rhizosphere soil fraction, and then centrifuged at

$1711,500 \mathrm{~g}$ for 20 mins. After centrifugation, the supernatant was discarded, and the pellet

172 immediately stored at $-80^{\circ} \mathrm{C}$. In the unplanted soil controls (i.e., the bulk soil pots), a portion of

173 soil corresponding to the area explored by roots was collected with a spatula and processed as

174 described for planted soils. Until DNA extraction, all the samples were stored at $-80^{\circ} \mathrm{C}$. 
176

177 Total DNA was extracted from the rhizosphere and unplanted soil samples using FastDNA ${ }^{\mathrm{TM}}$

178 SPIN kit for soil (MP Biomedicals, Solon, USA) following the instructions by the manufacturer.

179 To assess the concentration and the quality (260/280 $\mathrm{nm}$ and 260/230 $\mathrm{nm}$ ratios) of the extracted

180

181

182

183

184

185

186

187

188

189

190

191

192

193

194

195

196

197

198

Metagenomic DNA extraction from rhizosphere and bulk soil specimens

DNA, a Nanodrop 2000 spectrophotometer (Thermo Fisher Scientific, Waltham, USA) was used and samples were stored at $-20^{\circ} \mathrm{C}$ until further analysis. Aliquots at a final DNA concentration of $10 \mathrm{ng} \mathrm{mL}{ }^{-1}$ were prepared for each sample using sterilized deionized water, and stored at $-20^{\circ} \mathrm{C}$.

\section{S rRNA gene amplicon library construction}

The amplicon library was generated via a selective PCR amplification of the hypervariable V4 region of the $16 \mathrm{~S}$ rRNA gene using the PCR primers 515F (5'-GTGCCAGCMGCCGCGGTAA3') and 806R (5'- GGACTACHVGGGTWTCTAAT -3') as previously described (RobertsonAlbertyn et al., 2017; Alegria Terrazas et al., 2020). Briefly, PCR primer sequences were fused with Illumina flow cell adapter sequences at their 5 ' termini and the 806R primers contained 12mer unique 'barcode' sequences to enable the multiplexed sequencing of several samples in a single pool (Caporaso et al., 2012).

For each individual bulk and rhizosphere preparations, $50 \mathrm{ng}$ of DNA was subjected to PCR amplification using the Kapa HiFi HotStart PCR kit (Kapa Biosystems, Wilmington, USA). The individual PCR reactions were performed in $20 \mu \mathrm{L}$ final volume and contained:

- $4 \mu \mathrm{L}$ of $5 X$ Kapa HiFi Buffer

- $10 \mu \mathrm{g}$ Bovine Serum Albumin (BSA) (Roche, Mannheim, Germany)

- $\quad 0.6 \mu \mathrm{L}$ of a $10 \mathrm{mM}$ Kapa dNTPs solution

- $\quad 0.6 \mu \mathrm{L}$ of $10 \mu \mathrm{M}$ solutions of the $515 \mathrm{~F}$ and $806 \mathrm{R}$ PCR primers 
199

200

201

202

203

204

205

206

207

208

209

210

211

212

213

214

215

216

217

218

219

220

221

- $\quad 0.25 \mu \mathrm{L}$ of Kapa HiFi polymerase

Reactions were performed in a G-Storm GS1 thermal cycler (Gene Technologies, Somerton, UK) using the following programme: $94^{\circ} \mathrm{C}(3 \mathrm{~min})$, followed by 35 cycles of $98^{\circ} \mathrm{C}(30 \mathrm{~s}), 50^{\circ} \mathrm{C}$ (30 s) $72^{\circ} \mathrm{C}(1 \mathrm{~min})$ and a final step of $72^{\circ} \mathrm{C}(10 \mathrm{~min})$. For each $515 \mathrm{~F}-806 \mathrm{R}$ primer combination, a no template control (NTC) was subjected to the same process. To minimize potential biases originating during PCR amplifications, individual reactions were performed in triplicate and 2 independent sets of triplicate reactions per barcode were performed.

To check the amplification and/or any possible contamination, prior to purification, $6 \mu \mathrm{L}$ aliquots of individual replicates and the corresponding NTCs were inspected on 1.5\% agarose gel. Only samples that display the expected amplicon size and no detectable contamination in NTCs on gel were retained for library preparation.

Individual PCR amplicons replicates were then pooled in a single plate, moving each sample to a specific position according to their barcode. They were purified using Agencourt AMPure XP Kit (Beckman Coulter, Brea, USA) with $0.7 \mu \mathrm{L}$ AmPure XP beads per $1 \mu \mathrm{L}$ of sample. Following purification, $6 \mu \mathrm{L}$ of each sample was quantified using PicoGreen (Thermo Fisher Scientific, Watham, USA). Once quantified, individual barcode samples were pooled to a new tube in an equimolar ratio to generate amplicons libraries.

\section{Illumina $16 S$ rRNA gene amplicon sequencing}

Amplicon libraries were supplemented with $15 \%$ of a $4 \mathrm{pM}$ phiX solution and run at $10 \mathrm{pM}$ final concentration on an Illumina MiSeq system with paired end 2x 150 bp reads (Caporaso et al., 2012) as recommended, to generate FASTQ sequence files for processing and analysis. 
222

223

224

225

226

227

228

229

230

231

232

233

234

235

236

237

238

239

240

241

242

243 dataset.

\section{Amplicon sequencing reads processing}

Sequencing reads were processed using a customized bioinformatics pipeline as described before (Terrazas et al., 2019). Briefly, sequencing reads were subjected to quality assessment using FastQC (Andrews et al., 2015). Amplicon Sequencing Variants (ASVs) were then generated using DADA2 version 1.10 (Callahan et al., 2016) and R 3.5.2 (Team R Development Core, 2018) following the basic methodology outlined in the 'DADA2 Pipeline Tutorial (1.10)'. Read filtering was carried out using the DADA2 pairedFastqFilter method, trimming 10bp of sequence from the 5' of each reads using a truncQ parameter of 2 and maxEE of 2 . The remainder of the reads were of high quality so no 3' trimming was deemed necessary. The dada method was run to determine the error model with a MAX_CONSIST parameter of 20, following which the error model converged after 9 and 12 rounds for the forward and reverse reads respectively. The DADA2 method was then run with the resulting error model to denoise the reads using sample pooling, followed by read merging, using the default minOverlap parameter of 12 bases, then chimera removal using the consensus method. Taxonomy assignment was carried out using the RDP Naive Bayesian Classifier through the 'assign Taxonomy' method, with the SILVA database (Quast et al., 2012) version 138, using a minimum bootstrap confidence of 50. The DADA2 outputs were converted to a Phyloseq object (version 1.26.1) (McMurdie \& Holmes, 2013). ASVs assigned to chloroplast and mitochondria, putatively representing host contamination, as well as of ASVs previously identified as putative lab contaminant (Pietrangelo et al., 2018) were removed in silico from the original Phyloseq object. Likewise, ASVs lacking taxonomic classification at Phylum level (i.e., classified as "NAs") were removed from the 
244 As a secondary quality filtering approach (Bokulich et al., 2013), we applied an abundance

245 filtering for any given ASVs to be retained in the final dataset of 20 reads in at least $11 \%$ of the

246 samples, representing the number of replicates for a given one sample type/condition. In this

247 way, an ASV with overall low abundance due to its association with only one given condition

248 would have been retained and analysed in the dataset. Upon completion of this additional

249 filtering step, we retained 6615714 reads $(\min =25615 ; \max =305$ 408, mean $=147015.9)$

250 representing over $93.5 \%$ of the input quality filtered, non-contaminant sequences. Upon

251 completion of this additional filtering step, individual ASVs were agglomerated at Genus level in

252 Phyloseq. Finally, to control for sample-to-sample differences exceeding a factor of $\sim 10 \mathrm{X}$ in 253 sequencing depth (Weiss et al., 2017), we downsized the Phyloseq object at 25,000 reads per 254 sample.

255 Statistical analysis

256 Data analysis was performed in R software v 3.5.2. The following $\mathrm{R}$ packages were used:

257 Phyloseq v.1.26.1 (McMurdie \& Holmes, 2013) for Alpha and Beta-diversity indexes; DESeq2

258 v1.22.2 (Love, Huber \& Anders, 2014) for the differential analysis of microbial enrichment;

259 ggplot2 v.3.3.2 (Wickham, 2016) for data visualization; Vegan v.2.5-6 (Oksanen et al., 2019) for

260 statistical analysis of beta-diversity; PMCMR v.4.3 (Pohlert, 2018) for non-parametric analysis

261 of variance.

262 The analysis of the microbiota data was performed on filtered "Phyloseq object" described above 263 and linking into the analysis the mapping file (metadata information). For the Alpha-diversity

264 analysis, Chao1, Observed ASVs and Shannon indices were calculated using the function to 265 estimate richness included in the Phyloseq package. 
266 For the Beta-diversity analysis, the rarefied ASV table was used as input to compute a Bray-

267 Curtis, dissimilarity matrices. This dissimilarity matrix was visualized using Principal

268 Coordinates Analysis (PCoA) and Canonical Analysis of Principal coordinates (CAP) (Anderson

$269 \&$ Willis, 2003). Beta-diversity dissimilarity matrices were tested by Permutational Multivariate

270 Analysis of Variance (Permanova) using Adonis function in Vegan package over 5,000

271 permutations, to calculate the statistical significance.

272

273 Microbial enrichment analysis

274 The analysis of the microbial enrichment was performed using the DESeq2 package (Love,

275 Huber \& Anders, 2014), in order to identify the number of genera significantly enriched in pair-

276 wise comparisons with an adjusted $p$ value (False Discovery Rate, FDR $p<0.05$ ). The microbial

277 enrichment analysis was carried out between bulk soil and planted soil to evaluate the impact of

278 gramine on the 'rhizosphere effect'. The number of genera y enriched in the rhizosphere samples

279 subjected to the three different concentrations of gramine, in both Morex and Barke, was plotted

280 using the package UpSetR (Conway, Lex \& Gehlenborg, 2017). In parallel we performed a

281 series of pair-wise comparisons between Morex and Barke using the genera enriched in these

282 latter specimens from unplanted soil controls. Differentially enriched genera were visualized

283 using ternary plots as previously described (Bulgarelli et al., 2012). 


\section{Results}

285

286

287

288

289

290

291

292

293

294

295

296

297

298

299

300

301

302

303

304

305

306

307

\section{Gramine application impacts on the alpha- and beta-diversity properties of the microbiota} thriving at the barley root-soil interface

Gramine availability and mobility in Quarryfield soil are two prerequisites to fulfill its allelopathic ability towards target plants and inter-organismal relationships. Thus, we assessed its adsorption applying several two- and three-parameter non-linearized isotherms models, with the best fit based on the residual sum of squares (RSSs; Fig. S1). This choice was dictated by the fact that non-linear forms permit greater accuracy in predicting parameters compared to linear forms (Foo \& Hameed, 2010). The model with the best fit, the Sigmoidal Langmuir isotherm model, resulted in a sigmoidal curve, describing a cooperative adsorption phenomenon, which is common for non-polar compounds (Sparks, 2003; Limousin et al., 2007). A K $\mathrm{foc}_{\text {, defined as }}$ organic-carbon normalized Freundlich distribution coefficient, estimated at 1390 (OECD, 2001) classifies gramine as slightly mobile in this particular soil according to FAO Mobility Classification (FAO, 2000), yet remaining available in the soil solution. In addition, no significant differences were recorded by comparing plant dry weight at sampling time (Fig. S2, ANOVA followed by Tukey HSD test, Barke $p$ value $=0.432$ and Morex $p$ value $=0.9$ ). These data indicate that Quarryfield soil is a suitable substrate to investigate the impact of the exogenous application of gramine and the root-soil interface and that this latter is not associated to obvious pleiotropic effect on barley growth.

Next, we generate a 16S rRNA gene amplicon library from 45 bulk soil and rhizosphere samples exposed to different concentration of gramine. Upon processing of the sequencing reads in silico (Material and Methods) with a protocol comparable to previous studies conducted in the same 
308 soil type (Robertson-Albertyn et al., 2017; Alegria Terrazas et al., 2020), we failed to identify a 309 significant effect of the treatment on the alpha-diversity parameters of the tested communities

310 (Fig. 1, Kruskal-Wallis test followed by Dunn's post-hoc test, Observed genera $\mathrm{p}$ value $=0.5267$,

311 Chao1 $\mathrm{p}$ value $=0.6657$ and Shannon $\mathrm{p}$ value $=0.6002$, upon Bonferroni correction).

312 Conversely, we observed a clear impact of the external application of gramine on the bacterial

313 communities thriving at the root soil interface regardless of the applied concentration: both a

314 Canonical Analysis of Principal Coordinates (CAP, Fig. 2) and a Principal Coordinates Analysis

315 (PCoA, Fig. S3) built on a Bray-Curtis distance matrix revealed a partition of the microbiota

316 associated to the applied treatment. Of note, the effect of gramine appeared more pronounced on

317 bulk soil samples than rhizosphere specimens. Congruently, a permutational analysis of variance

318 computed on both matrices indicated a significant effect of the individual microhabitat, bulk soil,

319 Morex and Barke rhizosphere, respectively (R2 38.6\%, Adonis test $\mathrm{p}$ value $=0.00020 ; 5000$

320 permutations; Table 1) and the interaction term between gramine application and microhabitat

321 (R2 6.6; Adonis test $\mathrm{p}$ value $=0.01540 ; 5000$ permutations; Table 1$)$. Conversely, the

322 significance of the impact of gramine per se failed marginally to pass the significance threshold

323 imposed (R2 4.9\%; Adonis test $\mathrm{p}$ value $=0.05559 ; 5000$ permutations; Table 1$)$. Interestingly,

324 we obtained strikingly similar results when this calculation was performed using individual

325 ASVs (Fig. S4 and Fig. S5), suggesting that in the impact of gramine applications on the bacteria 326 proliferating at the root-soil interface is coded by taxonomically conserved portions of their 327 genomes.

328 
332 The observation that rhizosphere profiles tend to converge on the computed ordinations (see Fig.

3332 and Fig. S3), suggested that the two barley genotypes evolved the capacity of reverting, at least

334 in part, the selective pressure of gramine on soil bacteria. To quantify this phenomenon, we

335 implemented pair-wise comparisons between individual genera retrieved from unplanted soil and

336 rhizosphere communities at the three levels of gramine tested. Congruently with the initial

337 observation, we determined that the majority of genera enriched in the rhizosphere of either

338 genotype are comparable across gramine treatments (Fig. 3, Wald test, individual p values $<0.05$,

339 FDR corrected). Conversely, 18 genera, belonging to 16 distinct higher taxonomic ranks, whose

340 cumulative relative abundance represented $\sim 1.66 \%$ and $\sim 1.12 \%$ of the Morex and Barke

341 rhizosphere communities respectively, were identified as gramine-responsive in a genotype-

342 independent manner (Fig. 4, Wald test, individual p values $<0.05$, FDR corrected). Interestingly,

343 we noticed that the host genotype drives the enrichment of genera whose abundance in gramine-

344 treated bulk soil samples was almost obliterated. When looking at the taxonomic affiliation of

345 these rhizosphere- and gramine-responsive genera we observed a broad phylogenetic affiliation,

346 encompassing members of the phyla Acidobacteria, Actinobacteria, Bacteroidetes and

347 Proteobacteria. Next, we inspected how gramine application impacted on the genotype-driven

348 diversification of the rhizosphere bacterial microbiota, using the number of genera a) enriched

349 from soil and b) differentially enriched between the tested genotypes as a readout for this

350 analysis. This analysis revealed that plants exposed to no gramine or the lowest dosage displayed

351 a differential enrichment between cultivars of 31 and 42 genera, respectively (Fig. 5A and 5B,

352 Wald test, individual $\mathrm{p}$ values $<0.05$, FDR corrected). Conversely, at the highest gramine dosage

353 the host genotype effect was limited to 12 differentially enriched genera (Fig. 5C, Wald test,

354 individual $\mathrm{p}$ values $<0.05$, FDR corrected). When we inspected the taxonomic affiliation of these 
355 differentially enriched genera, we made two observations. First, members of the phylum

356 Proteobacteria accounted for the majority of differentially recruited genera (14 out of 31 genera

357 at gramine $0 \mu \mathrm{mol} \mathrm{L}^{-1}, 24$ out of 42 at gramine $24 \mu \mathrm{mol} \mathrm{L}^{-1}$ and 6 out of 12 at gramine $46 \mu \mathrm{mol}$

$358 \mathrm{~L}^{-1}$, respectively). The second observation was that despite the number of differentially regulated

359 genera at the intermediate dosage of gramine was comparable to mock-treated specimens, the

360 magnitude of the host selection was modulated by the host genotype itself, as Barke enriched for

361 almost three times the number of Proteobacteria upon gramine application.

362

363 Discussion

364

365 In this study we demonstrated that the exogenous application of the indole-alkaloid gramine

366 produced reproducible perturbations of the microbiota thriving at the barley root-soil interface

367 without triggering a discernable negative effect on plant growth performance on two barley elite

368 varieties.

369 The observation that gramine applications impact on the soil microbiota is aligned with the

370 findings of a recent survey performed by Schütz and co-workers monitoring the impact of the

371 application of several plant secondary metabolites, including gramine, on bacterial communities

372 of a German agricultural soil (Schütz et al., 2021). Similar to our findings, this study failed to

373 identify an effect of gramine on community richness (i.e., alpha diversity, Fig. 1), while

374 observing a shift in community composition (i.e., beta diversity, Fig. 2). Of note, the impact of

375 gramine applications on individual bacterial enrichments displayed an experimental effect: in our

376 investigation this was manifested with an apparent suppression of bacterial proliferation in

377 unplanted soil controls more pronounced than previously reported (Schütz et al., 2021). An

378 alternative, and not mutually exclusive scenario, is that gramine acts as a substrate for the growth 
379 of other or additional bacterial members of the soil biota capable of outperforming taxa identified

380 in this investigation.

381 In addition to differences in applications per se, it is important to consider that those experiments

382 were conducted using different soil types. For instance, soil $\mathrm{pH}$, one of the main drivers of

383 bacterial community composition in soil (Rousk et al., 2010), in the two studies differed of $\sim 0.5$

384 unit. Although this factor alone is unlikely to explain the differences between the studies, it may

385 represent a contributing factor, alongside other parameters such as organic matter, to the

386 differential impact of gramine applications on soil microbes. This concept is similar to what

387 observed for the exogenous application to grassland soils of low-molecular weight carbon

388 compounds, mimicking plants primary metabolites, altering microbiota composition in a soil-

389 and substrate-dependent manner (Eilers et al., 2010).

390

391 Studies conducted with the model plant Arabidopsis thaliana contributed to define of the impact 392 of secondary metabolites on the composition and function of the plant microbiota. For example,

393 the Brassicaceae-specific metabolites glucosinolates emerged as a key regulator of the outcome

394 of the symbiotic associations between A. thaliana and Colletotrichum tofieldiae, a fungal

395 member of the Arabidopsis microbiota (Hiruma et al., 2016). Likewise, in an elegant association

396 mapping study, Koprivova and colleagues identified a new nexus between a host genetic

397 diversity and microbiota functions (Koprivova et al., 2019). In particular, genes underpinning the

398 biosynthesis of the plant secondary metabolite camalexin emerged as regulators of sulfatase

399 activities of the microbiota and its plant probiotic potential (Koprivova et al., 2019).

400 
401 As A. thaliana is not a cultivated plant, we decided to compare the impact of gramine application

402 on barley-associated communities with the one of other secondary metabolites identified in

403 grasses such as maize, sorghum and oat which, similar to barley, have been exposed to the

404 processes of domestication and breeding selection.

405 For instance, we identified a limited, but significant, effect of gramine application on the

406 composition of the bacterial communities populating the root-soil interface (Fig. 3), indicating

407 that gramine per se (or lack thereof) does not disrupt the capacity of individual barley genotypes

408 of assembling a distinct rhizosphere microbiota. This is congruent with data gathered from

409 studies conducted using maize lines impaired in the biosynthesis of benzoxazinoids grown in

410 agricultural soils: despite mutants were capable of recruiting a distinct microbiota, this latter was

411 compositionally different from the one associated with wild type lines (Hu et al., 2018;

412 Kudjordjie et al., 2019). We consider these observations particularly relevant as benzoxazinoids

413 are secondary metabolites produced by several grasses (Frey et al., 2009) with the important

414 exception of the Hordeum vulgare clade (Grün, Frey \& Gierl, 2005). Similar to gramine,

415 benzoxazinoid display allelochemical, antimicrobial and insecticidal properties (Niemeyer \&

416 Perez, 1994; Niemeyer, 2009). A prediction of this observation is that, within the Poaceae

417 family, different classes of secondary metabolites may have evolved to fine-tune microbiota

418 composition. Congruently, sorghum produces a species-specific allelopathic compound

419 designated sorgoleone (Czarnota et al., 2001; Dayan et al., 2010) capable of selectively

420 modulating bacterial microbiota composition as demonstrated by experiments conducted using

421 RNA-interference lines impaired in sorgoleone biosynthesis grown under soil conditions (Wang

422 et al., 2021). Likewise, oat plants impaired in the production of avenacin, a triterpenoid

423 defensive compound active against fungal pathogens (Papadopoulou et al., 1999), recruit a 
424 taxonomically distinct rhizosphere microbiota compared to cognate wild type plants (Turner et

425 al., 2013). Interestingly, the effect of avenacin manifested predominantly at the level of the

426 eukaryotic component of the microbiota, particularly the protists Amoebozoa and Alveolata,

427 rather than the prokaryotic counterpart (Turner et al., 2013). Although differences in the

428 experimental and sequencing procedures existing among the aforementioned studies hinder the

429 capacity of establishing first principles, these observations suggest that, in cereals, species-

430 specific secondary metabolites act as a "gatekeepers" in the multi-step selection process

431 proposed for the diversification of the plant microbiota from the surrounding soil communities

432 (Bulgarelli et al., 2013; Edwards et al., 2015). The development of barley isogenic lines

433 contrasting for gramine biosynthesis will be required to overcome a limitation of our

434 investigation and ultimately prove (or disprove) these principles.

435

436 The impact of gramine application on the taxonomic composition of the barley rhizosphere

437 microbiota revealed a relatively broad phylogenetic impact: members of 16 prokaryotic orders

438 responded to gramine application in a dosage- and host genotype-independent manner (Fig. 4).

439 Among those, of particular interest is the order Nitrosotaleales represented by the Candidatus

440 genus Nitrosotalea. Member of this lineage have previously been characterized as ammonia-

441 oxidizing archaea, i.e., responsible for the rate-limiting step in the process of nitrification

442 (Treusch et al., 2005), and, despite being autotrophic organisms, are capable of differential

443 physiological responses in the presence of organic substrates (Lehtovirta-Morley et al., 2014).

444 For instance, our data indicate that the application of gramine to unplanted soil communities

445 suppress the proliferation of this member of ammonia oxidizing archaea, pointing at a role of this

446 compound in the biological inhibition of nitrification, as observed for others plant-derived 
447 compounds (Tesfamariam et al., 2014; Kaur-Bhambra et al., 2021). Yet, this scenario is difficult

448 to reconcile with the observation that Candidatus genus Nitrosotalea is enriched in the

449 rhizosphere of gramine-treated plants. A possible explanation could be derived by the niche

450 adaptation of ammonia oxidizing archaea: barley seedlings, similar to other grasses, have high a

451 rate of ammonia uptake and, in turn, this may create the optimum substrate conditions for the

452 proliferation of organisms like Candidatus genus Nitrosotalea despite the presence of putative

453 inhibitors (Thion et al., 2016). Congruently, previous experiments conducted with different

454 barley genotypes identified ammonia oxidizing archaea among members of the resident

455 rhizosphere microbiota in different soil types (Glaser et al., 2010). An alternative, but not

456 mutually exclusive, scenario is that the gramine inhibitory effect, is reduced by the activity of

457 other microorganisms in the rhizosphere compared to the unplanted soil control. As the

458 biological inhibition of nitrification has positive implications for sustainable crop production

459 (Coskun et al., 2017), it will be interesting to further investigate the relationships between

460 gramine biosynthesis and ammonia oxidation in prokaryotes.

461

462 Conversely, when we inspected the impact of applications in a genotype-dependent manner we

463 observed that the majority of microbes responding to gramine belong to the phylum

464 Proteobacteria (Fig. 5). Members of this phylum also respond to differential exudation of

465 benzoxazinoid (Jacoby, Koprivova \& Kopriva, 2021), possibly representing another element of

466 selection of cereal secondary metabolites towards the soil biota. As Proteobacteria represent the

467 most abundant members of the plant microbiota across host species (Hacquard et al., 2015), the

468 observed gramine effect may simply mirror the dominance of this group at the root-soil interface.

469 Yet, a recent study conducted on maize demonstrated that Proteobacteria, specifically members 
470 of the family Oxalobacteraceae, promote lateral root density and shoot dry weight (proxies for

471 plant growth) under nitrogen limiting conditions (Yu et al., 2021). As the soil tested in our

472 experiments is limited in the availability of nitrogen for barley growth (Terrazas et al., 2019) and

473 considering the enrichment of putative ammonia oxidizers archaea in the rhizosphere of treated

474 plants (see above), it can be speculated that the differential Proteobacterial enrichment triggered

475 by gramine application may be linked to nitrogen turnover in the rhizosphere. An additional

476 observation derived from these experiments is that gramine application triggers a differential

477 microbial recruitment in the two tested genotypes. A recent investigation conducted in tomato

478 revealed that a component of the root exudates can be induced by the exposure to a given

479 microbiota composition (Korenblum et al., 2020): it can therefore to be hypothesized that the

480 differential compositional differences observed upon gramine application may be the result of a,

481 genotype-dependent, fine-tuning of exudate profiles. An alternative, not mutually exclusive

482 scenario, is a differential rate of gramine degradation in the rhizosphere (Ghini, Burton \& Gros,

483 1991) of the two genotypes as this would alter the bioavailability of this compound to the

484 resident members of the microbiota. Regardless of the scenario, it is interesting to note that

485 gramine applications failed to trigger a sustained enrichment of members of the Actinobacteria,

486 which can be considered as a hallmark of elite, gramine-free, barley genotypes grown in the

487 same soil type (Alegria Terrazas et al., 2020) and in other modern/ancestral plant pairs (Pérez-

488 Jaramillo et al., 2018). 
490

491

492

493

494

495

496

497

498

499

500

501

502

503

504

505

506

507

508

509

510

511

512

513

514

515 Figure legend

516

\section{Conclusions} interactions.

\section{Data availability}

Our results indicate that the application of the indole-alkaloid gramine modulates the proliferation of a subset of soil microbes with relatively broad phylogenetic assignments. This effect is two-pronged: a component of the barley microbiota responds to gramine application in a genotype- and dosage-independent manner while other or additional host-derived mechanisms, underpinning the genotype diversification in the rhizosphere, modulate the effect of gramine application with a bias for members of the phylum Proteobacteria. As gramine biosynthesis has previously been reported as stress induced (Velozo et al., 1999; Matsuo et al., 2001), we anticipate that exposure to different soil characteristics, including different microbiomes, is likely to amplify (or obliterate) the effect of this metabolite on edaphic microbes. A limitation of our investigation was represented by the lack of isogenic lines contrasting for gramine biosynthesis. We therefore propose to capitalise on these initial observations, and the expanding genomic resources for barley (Maurer et al., 2015; Jayakodi et al., 2020), to resolve the genetic basis of gramine biosynthesis and ultimately elucidate its adaptive value for plant-microbe

The sequences generated in the 16S rRNA gene sequencing survey are deposited in the European Nucleotide Archive (ENA) under the accession number PRJEB39836. The version of the individual packages and scripts used to analyse the data and generate the figures of this study are available at https://github.com/Stramon1um/gramine_microbiome. 
517 Figure 1. Boxplot of alpha diversity indexes. Alpha diversity Observed genera, Chao1 and

518 Shannon indexes, of Bulk soil, Barke and Morex, at three different gramine concentrations.

519 Individual dots depict individual biological replicates; no significant differences observed for

520 gramine treatment upon Kruskal-Wallis test followed by Dunn's post-hoc test, individual p

521 values $>0.05$, Bonferroni corrected.

522

523

524

Figure 2. Canonical analysis of Principal Coordinates (CAP) constructed on a Bray-Curtis

525

526

527

528

529

530

531

532

533

534

535

536

537

538

539

540

541

542

543

544

545

546

dissimilarity matrix of Bulk soil, Barke and Morex, at three different gramine concentrations. Individual shapes depict individual samples, color-coded according the gramine treatment imposed on them. The ordination was constrained for genotype and gramine concentrations.

Figure 3. Gramine modulates bacterial abundances at the barley root-soil interface UpSetR plots of Genera significantly enriched in and differentiating the rhizosphere of A) Morex or B) Barke from unplanted soil, at the three levels of gramine tested. Vertical bars denote the number of genera enriched shared or unique for each comparison, while the horizontal bars the number of genera enriched in the indicated gramine concentration. In A and B genera differentially enriched at individual $\mathrm{p}$ values $<0.05$, Wald Test, FDR corrected.

Figure 4. Taxonomy and abundances of the gramine-responsive genera. Heatmap of the 18 bacterial genera, classified either at Order or Phylum level, significantly enriched in rhizosphere samples in a genotype- and gramine dosage- (24 and $46 \mu \mathrm{M}$, respectively) independent manner. Microbial enrichments defined at individual $p$ values $<0.05$, Wald Test, FDR corrected.

\section{Figure 5. Gramine application attenuates the genotype effect on the rhizosphere microbiota}

Ternary plots depicting bacteria distribution across the indicated microhabitats in sample exposed to A) no gramine, B) gramine $24 \mu \mathrm{M}$ or C) gramine $46 \mu \mathrm{M}$. In each plot, individual dots depict individual microbes whose size is proportional their sequencing abundances. Position of the dots within the plots reflects the contribution of each microhabitats to microbial abundances. Coloured dots denote genera differentially enriched between Morex and Barke (individual p values $<0.05$, Wald Test, FDR corrected) color-coded according to their taxonomic affiliation at 
547 phylum level. Gray dots depict genera not enriched in the rhizosphere and/or not differentially 548 assembled between genotypes.

549

550

551

552

553

554

555

556

557

558

559

560

561

562

563

564

565

566

567

568

569

570

571

572

573

574

575

576

577

Table 1. Permutational analysis of rhizosphere microbiota variance computed on Bray-Curtis matrix, variance explained by the indicated variables and corresponding statistical significance.

Figure S1. Non-linearized isotherm adsorption models of the alkaloid gramine in Quarryfield soil. Adsorption isotherms of gramine in Quarryfield soil were fitted applying several nonlinear models: the two-parameter Langmuir and Freundlich isotherms and the three-parameter Sigmoidal Langmuir, Redlich-Peterson and Sips isotherms, along with their corresponding Residual Sum of Squares (RSSs) indicating the fitness of the model to the data. $\mathrm{Ce}=$ gramine concentration at equilibrium, $\mathrm{qe}=$ gramine concentration adsorbed on the soil.

Figure S2. Above-ground performance of the tested genotypes. Stem dry weight of Barke and Morex barley plants subjected to different gramine concentrations at the time of sampling. No significant differences using ANOVA followed by Tukey HSD test, $p$ value $>0.05$

Figure S3. Principal Coordinates Analysis (PCoA) constructed on a Bray-Curtis dissimilarity matrix of Bulk soil, Barke and Morex, at three different gramine concentrations. Individual shapes depict individual samples, color-coded according the gramine treatment imposed on them. The ordination was constrained for genotype and gramine concentrations.

Figure S4. Alpha diversity computed at Amplicon Sequencing Variants level. Alpha diversity Observed ASVs, Chao1 and Shannon indexes, of Bulk soil, Barke and Morex, at three different gramine concentrations. Individual dots depict individual biological replicates; no significant differences observed.

Figure S5. CAP Bray-Curtis computed at Amplicon Sequencing Variants level. Individual shapes depict individual samples, color-coded according the gramine treatment imposed on them. The ordination was constrained for genotype and gramine concentrations.

Peer) reviewing PDF | (2021:07:64033:1:0:NEW 5 Oct 2021) 
578

579

580 


\section{Funding}

582

583 The work presented in this manuscript was supported by a Royal Society of Edinburgh/Scottish

584 Government Personal Research Fellowship co-funded by Marie Curie Actions and a UK

585 Research and Innovation grant (BB/S002871/1) awarded to DB.

586

587 Acknowledgement

588

589 We thank Malcolm Macaulay, (The James Hutton Institute, UK) for the technical assistance in 590 preparing the amplicon sequencing library and Rodrigo Alegria Terrazas (Mohammed VI

591 Polytechnic University, Morocco) for the critical comments on the manuscript.

592

593 


\section{References}

595 Åhman I, Tuvesson S, Johansson M. 2000. Does indole alkaloid gramine confer resistance in

596

597

598

599

600

601

602

603

604

605

606

607

608

609

610

611

612

613

614

615

616

617

618

619

620

621

622

623

624

625

626

627

628

629

630

631

632

633

634

635

636

637

638

639 barley to aphid Rhopalosiphum padi? Journal of chemical ecology 26:233-255. DOI: 10.1023/A:1005405915031.

Alegria Terrazas R, Balbirnie-Cumming K, Morris J, Hedley PE, Russell J, Paterson E, Baggs EM, Fridman E, Bulgarelli D. 2020. A footprint of plant eco-geographic adaptation on the composition of the barley rhizosphere bacterial microbiota. Scientific Reports 10:1-14. DOI: $10.1038 / \mathrm{s} 41598-020-69672-\mathrm{x}$.

Alegria Terrazas R, Giles C, Paterson E, Robertson-Albertyn S, Cesco S, Mimmo T, Pii Y, Bulgarelli D. 2016. Plant-microbiota interactions as a driver of the mineral Turnover in the Rhizosphere. Elsevier Ltd. DOI: 10.1016/bs.aambs.2016.03.001.

Anderson MJ, Willis TJ. 2003. Canonical analysis of principal coordinates: A useful method of constrained ordination for ecology. Ecology 84:511-525. DOI: 10.1890/00129658(2003)084[0511:CAOPCA]2.0.CO;2.

Andrews S, Krueger F, Seconds-Pichon A, Biggins F, Wingett S. 2015. FastQC. A quality control tool for high throughput sequence data. Babraham Bioinformatics. Babraham Institute 1:1.

Bokulich NA, Subramanian S, Faith JJ, Gevers D, Gordon JI, Knight R, Mills DA, Caporaso JG. 2013. Quality-filtering vastly improves diversity estimates from Illumina amplicon sequencing. Nature Methods 10:57-59. DOI: 10.1038/nmeth.2276.

Bulgarelli D, Garrido-Oter R, Münch PC, Weiman A, Dröge J, Pan Y, McHardy AC, SchulzeLefert P. 2015. Structure and function of the bacterial root microbiota in wild and domesticated barley. Cell Host and Microbe 17:392-403. DOI: 10.1016/j.chom.2015.01.011.

Bulgarelli D, Rott M, Schlaeppi K, Ver Loren van Themaat E, Ahmadinejad N, Assenza F, Rauf P, Huettel B, Reinhardt R, Schmelzer E, Peplies J, Gloeckner FO, Amann R, Eickhorst T, Schulze-Lefert P. 2012. Revealing structure and assembly cues for Arabidopsis rootinhabiting bacterial microbiota. Nature 488:91-5. DOI: 10.1038/nature11336.

Bulgarelli D, Schlaeppi K, Spaepen S, Ver Loren van Themaat E, Schulze-Lefert P. 2013. Structure and functions of the bacterial microbiota of plants. Annual review of plant biology 64:807-38. DOI: 10.1146/annurev-arplant-050312-120106.

Cai QN, Han Y, Cao YZ, Hu Y, Zhao X, Bi JL. 2009. Detoxification of gramine by the cereal aphid sitobion avenae. Journal of Chemical Ecology 35:320-325. DOI: 10.1007/s10886009-9603-y.

Callahan BJ, McMurdie PJ, Rosen MJ, Han AW, Johnson AJA, Holmes SP. 2016. DADA2: High-resolution sample inference from Illumina amplicon data. Nature Methods 13:581583. DOI: $10.1038 /$ nmeth.3869.

Canarini A, Kaiser C, Merchant A, Richter A, Wanek W. 2019. Root exudation of primary metabolites: Mechanisms and their roles in plant responses to environmental stimuli. Frontiers in Plant Science 10:157. DOI: 10.3389/fpls.2019.00157.

Caporaso JG, Lauber CL, Walters WA, Berg-Lyons D, Huntley J, Fierer N, Owens SM, Betley J, Fraser L, Bauer M, Gormley N, Gilbert JA, Smith G, Knight R. 2012. Ultra-highthroughput microbial community analysis on the Illumina HiSeq and MiSeq platforms. ISME Journal 6:1621-1624. DOI: 10.1038/ismej.2012.8.

Conway JR, Lex A, Gehlenborg N. 2017. UpSetR: An R package for the visualization of intersecting sets and their properties. Bioinformatics 33:2938-2940. DOI:

PeerJ reviewing PDF | (2021:07:64033:1:0:NEW 5 Oct 2021) 
640

641

642

643

644

645

646

647

648

649

650

651

652

653

654

655

656

657

658

659

660

661

662

663

664

665

666

667

668

669

670

671

672

673

674

675

676

677

678

679

680

681

682

683

684

685

10.1093/bioinformatics/btx364.

Corcuera LJ. 1993. Biochemical basis for the resistance of barley to aphids. Phytochemistry 33:741-747. DOI: 10.1016/0031-9422(93)85267-U.

Coskun D, Britto DT, Shi W, Kronzucker HJ. 2017. Nitrogen transformations in modern agriculture and the role of biological nitrification inhibition. Nature Plants 3:1-10. DOI: 10.1038/nplants.2017.74.

Czarnota MA, Paul RN, Dayan FE, Nimbal CI, Weston LA. 2001. Mode of Action, Localization of Production, Chemical Nature, and Activity of Sorgoleone: A Potent PSII Inhibitor in Sorghum spp. Root Exudates 1. Weed Technology 15:813-825. DOI: 10.1614/0890037x(2001)015[0813:moalop]2.0.co;2.

Dakora FD, Phillips DA. 2002. Root exudates as mediators of mineral acquisition in low-nutrient environments. In: Food Security in Nutrient-Stressed Environments: Exploiting Plants' Genetic Capabilities. Springer Netherlands, 201-213. DOI: 10.1007/978-94-017-1570623.

Dayan FE, Rimando AM, Pan Z, Baerson SR, Gimsing AL, Duke SO. 2010. Sorgoleone. Phytochemistry 71:1032-1039. DOI: 10.1016/j.phytochem.2010.03.011.

Edwards J, Johnson C, Santos-Medellín C, Lurie E, Podishetty NK, Bhatnagar S, Eisen JA, Sundaresan V, Jeffery LD. 2015. Structure, variation, and assembly of the root-associated microbiomes of rice. Proceedings of the National Academy of Sciences of the United States of America 112:E911-E920. DOI: 10.1073/pnas.1414592112.

Eilers KG, Lauber CL, Knight R, Fierer N. 2010. Shifts in bacterial community structure associated with inputs of low molecular weight carbon compounds to soil. Soil Biology and Biochemistry 42:896-903. DOI: 10.1016/j.soilbio.2010.02.003.

Escudero-Martinez C, Bulgarelli D. 2019. Tracing the evolutionary routes of plant-microbiota interactions. Current Opinion in Microbiology 49:34-40. DOI: 10.1016/j.mib.2019.09.013.

FAO. 2000. FAO PESTICIDE DISPOSAL SERIES 8 Assessing soil contamination A reference manual.

Foo KY, Hameed BH. 2010. Insights into the modeling of adsorption isotherm systems. Chemical Engineering Journal 156:2-10. DOI: 10.1016/J.CEJ.2009.09.013.

Frey M, Schullehner K, Dick R, Fiesselmann A, Gierl A. 2009. Benzoxazinoid biosynthesis, a model for evolution of secondary metabolic pathways in plants. Phytochemistry 70:16451651. DOI: 10.1016/j.phytochem.2009.05.012.

Ghini AA, Burton G, Gros EG. 1991. Biodegradation of the indolic system of gramine in Hordeum vulgare. Phytochemistry 30:779-784. DOI: 10.1016/0031-9422(91)85251-T.

Glaser K, Hackl E, Inselsbacher E, Strauss J, Wanek W, Zechmeister-Boltenstern S, Sessitsch A. 2010. Dynamics of ammonia-oxidizing communities in barley-planted bulk soil and rhizosphere following nitrate and ammonium fertilizer amendment. FEMS Microbiology Ecology 74:575-591. DOI: 10.1111/j.1574-6941.2010.00970.x.

Grün S, Frey M, Gierl A. 2005. Evolution of the indole alkaloid biosynthesis in the genus Hordeum: Distribution of gramine and DIBOA and isolation of the benzoxazinoid biosynthesis genes from Hordeum lechleri. Phytochemistry 66:1264-1272. DOI: 10.1016/j.phytochem.2005.01.024.

Hacquard S, Garrido-Oter R, González A, Spaepen S, Ackermann G, Lebeis S, McHardy AC, Dangl JL, Knight R, Ley R, Schulze-Lefert P. 2015. Microbiota and host nutrition across plant and animal kingdoms. Cell Host and Microbe 17:603-616. DOI: 10.1016/j.chom.2015.04.009. 
686 Hiruma K, Gerlach N, Sacristán S, Nakano RT, Hacquard S, Kracher B, Neumann U, Ramírez

687

688

689

690

691

692

693

694

695

696

697

698

699

700

701

702

703

704

705

706

707

708

709

710

711

712

713

714

715

716

717

718

719

720

721

722

723

724

725

726

727

728

729

730

731

D, Bucher M, O’Connell RJ, Schulze-Lefert P. 2016. Root Endophyte Colletotrichum tofieldiae Confers Plant Fitness Benefits that Are Phosphate Status Dependent. Cell 165:464-474. DOI: 10.1016/j.cell.2016.02.028.

Hu L, Robert CAM, Cadot S, Zhang X, Ye M, Li B, Manzo D, Chervet N, Steinger T, van der Heijden MGA, Schlaeppi K, Erb M. 2018. Root exudate metabolites drive plant-soil feedbacks on growth and defense by shaping the rhizosphere microbiota. Nature Communications 9:2738. DOI: 10.1038/s41467-018-05122-7.

Jacoby RP, Koprivova A, Kopriva S. 2021. Pinpointing secondary metabolites that shape the composition and function of the plant microbiome. Journal of Experimental Botany 72:5769. DOI: $10.1093 / \mathrm{jxb} / \mathrm{eraa} 424$.

Jayakodi M, Padmarasu S, Haberer G, Bonthala VS, Gundlach H, Monat C, Lux T, Kamal N, Lang D, Himmelbach A, Ens J, Zhang X-Q, Angessa TT, Zhou G, Tan C, Hill C, Wang P, Schreiber M, Fiebig A, Budak H, Xu D, Zhang J, Wang C, Guo G, Zhang G, Mochida K, Hirayama T, Sato K, Chalmers KJ, Langridge P, Waugh R, Pozniak CJ, Scholz U, Mayer KFX, Spannagl M, Li C, Mascher M, Stein N. 2020. The barley pan-genome reveals the hidden legacy of mutation breeding. DOI: 10.1038/s41586-020-2947-8.

Jones DL, Nguyen C, Finlay RD. 2009. Carbon flow in the rhizosphere: Carbon trading at the soil-root interface. Plant and Soil 321:5-33. DOI: 10.1007/s11104-009-9925-0.

Kaur-Bhambra J, Wardak DLR, Prosser JI, Gubry-Rangin C. 2021. Revisiting plant biological nitrification inhibition efficiency using multiple archaeal and bacterial ammonia-oxidising cultures. Biology and Fertility of Soils. DOI: 10.1007/s00374-020-01533-1.

Koprivova A, Schuck S, Jacoby RP, Klinkhammer I, Welter B, Leson L, Martyn A, Nauen J, Grabenhorst N, Mandelkow JF, Zuccaro A, Zeier J, Kopriva S. 2019. Root-specific camalexin biosynthesis controls the plant growth-promoting effects of multiple bacterial strains. Proceedings of the National Academy of Sciences of the United States of America 116:15735-15744. DOI: 10.1073/pnas.1818604116.

Korenblum E, Dong Y, Szymanski J, Panda S, Jozwiak A, Massalha H, Meir S, Rogachev I, Aharoni A. 2020. Rhizosphere microbiome mediates systemic root metabolite exudation by root-to-root signaling. Proceedings of the National Academy of Sciences of the United States of America 117:3874-3883. DOI: 10.1073/pnas.1912130117.

Kudjordjie EN, Sapkota R, Steffensen SK, Fomsgaard IS, Nicolaisen M. 2019. Maize synthesized benzoxazinoids affect the host associated microbiome. Microbiome 7:1-17. DOI: 10.1186/s40168-019-0677-7.

Larsson KAE, Zetterlund I, Delp G, Jonsson LM V. 2006. N-Methyltransferase involved in gramine biosynthesis in barley: Cloning and characterization. Phytochemistry 67:20022008. DOI: 10.1016/j.phytochem.2006.06.036.

Lebeis SL, Paredes SH, Lundberg DS, Breakfield N, Gehring J, McDonald M, Malfatti S, Del Rio TG, Jones CD, Tringe SG, Dangl JL. 2015. Salicylic acid modulates colonization of the root microbiome by specific bacterial taxa. Science 349:860-864. DOI: 10.1126/science.aaa8764.

Lehtovirta-Morley LE, Ge C, Ross J, Yao H, Nicol GW, Prosser JI. 2014. Characterisation of terrestrial acidophilic archaeal ammonia oxidisers and their inhibition and stimulation by organic compounds. FEMS Microbiology Ecology 89:542-552. DOI: 10.1111/15746941.12353.

Limousin G, Gaudet JP, Charlet L, Szenknect S, Barthès V, Krimissa M. 2007. Sorption

PeerJ reviewing PDF | (2021:07:64033:1:0:NEW 5 Oct 2021) 
732

733

734

735

736

737

738

739

740

741

742

743

744

745

746

747

748

749

750

751

752

753

754

755

756

757

758

759

760

761

762

763

764

765

766

767

768

769

770

771

772

773

774

775

776

777

isotherms: A review on physical bases, modeling and measurement. Applied Geochemistry 22:249-275. DOI: 10.1016/j.apgeochem.2006.09.010.

Love MI, Huber W, Anders S. 2014. Moderated estimation of fold change and dispersion for RNA-seq data with DESeq2. Genome Biology 15:1-21. DOI: 10.1186/s13059-014-0550-8.

Lugtenberg B, Kamilova F. 2009. Plant-growth-promoting rhizobacteria. Annual Review of Microbiology 63:541-556. DOI: 10.1146/annurev.micro.62.081307.162918.

Macaulay M, Ramsay L, Åhman I. 2020. Quantitative trait locus for resistance to the aphid Rhopalosiphum padi L. in barley (Hordeum vulgare L.) is not linked with a genomic region for gramine concentration. Arthropod-Plant Interactions 14:57-65. DOI: 10.1007/s11829019-09727-7.

Matsuo H, Taniguchi K, Hiramoto T, Yamada T, Ichinose Y, Toyoda K, Takeda K, Shiraishi T. 2001. Gramine increase associated with rapid and transient systemic resistance in barley seedlings induced by mechanical and biological stresses. Plant \& cell physiology 42:110311.

Maurer A, Draba V, Jiang Y, Schnaithmann F, Sharma R, Schumann E, Kilian B, Reif JC, Pillen K. 2015. Modelling the genetic architecture of flowering time control in barley through nested association mapping. BMC Genomics 16:290. DOI: 10.1186/s12864-015-1459-7.

Maver M, Miras-Moreno B, Lucini L, Trevisan M, Pii Y, Cesco S, Mimmo T. 2020. New insights in the allelopathic traits of different barley genotypes: Middle Eastern and Tibetan wild-relative accessions vs. cultivated modern barley. PLOS ONE 15:1-18. DOI: 10.1371/journal.pone.0231976.

McMurdie PJ, Holmes S. 2013. Phyloseq: An R Package for Reproducible Interactive Analysis and Graphics of Microbiome Census Data. PLoS ONE 8. DOI: 10.1371/journal.pone.0061217.

Moharramipour S, Takeda K, Sato K, Yoshida H, Tsumuki H. 1999. Inheritance of gramine content in barley. Euphytica 106:181-185. DOI: 10.1023/A:1003535823329.

Niemeyer HM. 2009. Hydroxamic acids derived from 2-hydroxy-2h-1,4-benzoxazin-3(4h)-one: Key defense chemicals of cereals. Journal of Agricultural and Food Chemistry 57:16771695. DOI: 10.1021/jf8034034.

Niemeyer HM, Perez FJ. 1994. Potential of Hydroxamic Acids in the Control of Cereal Pests, Diseases, and Weeds. In: 260-270. DOI: 10.1021/bk-1995-0582.ch019.

OECD. 2001. OECD Guidline for testing chemicals Estimation of the Adsorption Coefficient ( $K$ oc ) on Soil and on Sewage Sludge using High Performance Liquid Chromatography (HPLC) 121.

Oksanen AJ, Blanchet FG, Friendly M, Kindt R, Legendre P, Mcglinn D, Minchin PR, Hara RBO, Simpson GL, Solymos P, Stevens MHH, Szoecs E. 2019. Package 'vegan .'

Papadopoulou K, Melton RE, Leggett M, Daniels MJ, Osbourn AE. 1999. Compromised disease resistance in saponin-deficient plants. Proceedings of the National Academy of Sciences of the United States of America 96:12923-12928. DOI: 10.1073/pnas.96.22.12923.

Pascale A, Proietti S, Pantelides IS, Stringlis IA. 2020. Modulation of the Root Microbiome by Plant Molecules: The Basis for Targeted Disease Suppression and Plant Growth Promotion. Frontiers in Plant Science 10:1-23. DOI: 10.3389/fpls.2019.01741.

Pérez-Jaramillo JE, Carrión VJ, Bosse M, Ferrão LFV, De Hollander M, Garcia AAF, Ramírez CA, Mendes R, Raaijmakers JM. 2017. Linking rhizosphere microbiome composition of wild and domesticated Phaseolus vulgaris to genotypic and root phenotypic traits. ISME Journal 11:2244-2257. DOI: 10.1038/ismej.2017.85. 
778

779

780

781

782

783

784

785

786

787

788

789

790

791

792

793

794

795

796

797

798

799

800

801

802

803

804

805

806

807

808

809

810

811

812

813

814

815

816

817

818

819

820

821

822

823

Pérez-Jaramillo JE, Carrión VJ, de Hollander M, Raaijmakers JM. 2018. The wild side of plant microbiomes. Microbiome 6:143. DOI: 10.1186/s40168-018-0519-z.

Pérez-Jaramillo JE, Mendes R, Raaijmakers JM. 2016. Impact of plant domestication on rhizosphere microbiome assembly and functions. Plant Molecular Biology 90:635-644. DOI: $10.1007 / \mathrm{s} 11103-015-0337-7$.

Pietrangelo L, Bucci A, Maiuro L, Bulgarelli D, Naclerio G. 2018. Unraveling the composition of the root-associated bacterial microbiota of Phragmites australis and Typha latifolia. Frontiers in Microbiology 9:1-13. DOI: 10.3389/fmicb.2018.01650.

Pohlert T. 2018. Package 'PMCMRplus': Calculate Pairwise Multiple Comparisons of Mean Rank Sums Extended. :1-8. DOI: 10.18637/jss.v080.i01>.

Preece C, Peñuelas J. 2020. A Return to the Wild: Root Exudates and Food Security. Trends in Plant Science 25:14-21. DOI: 10.1016/j.tplants.2019.09.010.

Purugganan MD, Fuller DQ. 2009. The nature of selection during plant domestication. Nature 457:843-848. DOI: 10.1038/nature07895.

Quast C, Pruesse E, Yilmaz P, Gerken J, Schweer T, Yarza P, Peplies J, Glöckner FO. 2012. The SILVA ribosomal RNA gene database project: improved data processing and web-based tools. Nucleic acids research 41:D590--D596.

Robertson-Albertyn S, Alegria Terrazas R, Balbirnie K, Blank M, Janiak A, Szarejko I, Chmielewska B, Karcz J, Morris J, Hedley PE, George TS, Bulgarelli D. 2017. Root Hair Mutations Displace the Barley Rhizosphere Microbiota. Frontiers in Plant Science 8:1094. DOI: $10.3389 /$ fpls.2017.01094.

Rolfe SA, Griffiths J, Ton J. 2019. Crying out for help with root exudates: adaptive mechanisms by which stressed plants assemble health-promoting soil microbiomes. Current Opinion in Microbiology 49:73-82. DOI: 10.1016/j.mib.2019.10.003.

Rousk J, Bååth E, Brookes PC, Lauber CL, Lozupone C, Caporaso JG, Knight R, Fierer N. 2010. Soil bacterial and fungal communities across a $\mathrm{pH}$ gradient in an arable soil. ISME Journal 4:1340-1351. DOI: 10.1038/ismej.2010.58.

Schlaeppi K, Bulgarelli D. 2015. The Plant Microbiome at Work. Molecular Plant-Microbe Interactions 28:212-217. DOI: 10.1094/MPMI-10-14-0334-FI.

Schütz V, Frindte K, Cui J, Zhang P, Hacquard S, Schulze-Lefert P, Knief C, Schulz M, Dörmann P. 2021. Differential Impact of Plant Secondary Metabolites on the Soil Microbiota. Frontiers in Microbiology 12:666010. DOI: 10.3389/fmicb.2021.666010. Sepulveda BA, Corcuera LJ. 1990. Effect of gramine on the susceptibility of barley leaves to Pseudomonas syringae. Phytochemistry 29:465-467. DOI: 10.1016/0031-9422(90)85098-Z. Sparks DL. 2003. Sorption Phenomena on Soils. Environmental Soil Chemistry:133-186. DOI: 10.1016/b978-012656446-4/50005-0.

Sun XQ, Zhang MX, Yu JY, Jin Y, Ling B, Du JP, Li GH, Qin QM, Cai QN. 2013. Glutathione S-Transferase of Brown Planthoppers (Nilaparvata lugens) Is Essential for Their Adaptation to Gramine-Containing Host Plants. PLoS ONE 8. DOI: 10.1371/journal.pone.0064026.

Team R Development Core. 2018. A Language and Environment for Statistical Computing. $R$ Foundation for Statistical Computing 2:https://www.R-project.org.

Terrazas RA, Robertson-Albertyn S, Corral AM, Escudero-Martinez C, Balbirnie-Cumming K, Morris J, Hedley P, Paterson E, Baggs E, Abbott J, Bulgarelli D. 2019. Nitrogen availability modulates the host control of the barley rhizosphere microbiota. bioRxiv:605204. DOI: $10.1101 / 605204$.

Tesfamariam T, Yoshinaga H, Deshpande SP, Srinivasa Rao P, Sahrawat KL, Ando Y, Nakahara

Peer] reviewing PDF | (2021:07:64033:1:0:NEW 5 Oct 2021) 
824

825

826

827

828

829

830

831

832

833

834

835

836

837

838

839

840

841

842

843

844

845

846

847

848

849

850

851

852

853

854

855

856

857

858

859

860
K, Hash CT, Subbarao G V. 2014. Biological nitrification inhibition in sorghum: The role of sorgoleone production. Plant and Soil 379:325-335. DOI: 10.1007/s11104-014-2075-z.

The International Barley Genome Sequencing Consortium. 2012. A physical, genetic and functional sequence assembly of the barley genome. Nature 491:711-716.

Thion CE, Poirel JD, Cornulier T, De Vries FT, Bardgett RD, Prosser JI. 2016. Plant nitrogenuse strategy as a driver of rhizosphere archaeal and bacterial ammonia oxidiser abundance. FEMS Microbiology Ecology 92:91. DOI: 10.1093/femsec/fiw091.

Tottman DR, Makepeace RJ, Broad H. 1979. An explanation of the decimal code for the growth stages of cereals, with illustrations. Annals of Applied Biology 93:221-234. DOI: 10.1111/j.1744-7348.1979.tb06534.x.

Treusch AH, Leininger S, Kietzin A, Schuster SC, Klenk HP, Schleper C. 2005. Novel genes for nitrite reductase and Amo-related proteins indicate a role of uncultivated mesophilic crenarchaeota in nitrogen cycling. Environmental Microbiology 7:1985-1995. DOI: 10.1111/j.1462-2920.2005.00906.x.

Turner TR, James EK, Poole PS. 2013. The plant microbiome. Genome Biology 14. DOI: 10.1186/gb-2013-14-6-209.

Turner TR, Ramakrishnan K, Walshaw J, Heavens D, Alston M, Swarbreck D, Osbourn A, Grant A, Poole PS. 2013. Comparative metatranscriptomics reveals kingdom level changes in the rhizosphere microbiome of plants. ISME Journal 7:2248-2258. DOI: 10.1038/ismej.2013.119.

Velozo JA, Alvarez RI, Wächter GA, Timmermann BN, Corcuera LJ. 1999. Increase in gramine content in barley infested by the aphid Schizaphis graminum R. Phytochemistry 52:10591061. DOI: 10.1016/S0031-9422(99)00358-1.

Wang P, Chai YN, Roston R, Dayan FE, Schachtman DP. 2021. The Sorghum bicolor Root Exudate Sorgoleone Shapes Bacterial Communities and Delays Network Formation. mSystems 6. DOI: 10.1128/msystems.00749-20.

Weiss S, Xu ZZ, Peddada S, Amir A, Bittinger K, Gonzalez A, Lozupone C, Zaneveld JR, Vázquez-Baeza Y, Birmingham A, Hyde ER, Knight R. 2017. Normalization and microbial differential abundance strategies depend upon data characteristics. Microbiome 5:1-18. DOI: 10.1186/s40168-017-0237-y.

Wickham H. 2016. Ggplot2 : elegant graphics for data analysis. Springer.

Yu P, He X, Baer M, Beirinckx S, Tian T, Moya YAT, Zhang X, Deichmann M, Frey FP, Bresgen V, Li C, Razavi BS, Schaaf G, von Wirén N, Su Z, Bucher M, Tsuda K, Goormachtig S, Chen X, Hochholdinger F. 2021. Plant flavones enrich rhizosphere Oxalobacteraceae to improve maize performance under nitrogen deprivation. Nature Plants 7:481-499. DOI: 10.1038/s41477-021-00897-y. 


\section{Table $\mathbf{1}$ (on next page)}

Table 1- Permutational analysis of rhizosphere microbiota variance computed on BrayCurtis matrix

variance explained by the indicated variables and corresponding statistical significance. 


\section{Table 1:}

2

3 Permutational analysis of rhizosphere microbiota variance computed on Bray-Curtis matrix, variance 4 explained by the indicated variables and corresponding statistical significance. 5

\begin{tabular}{|c|c|c|}
\hline Factor & $\mathbf{R 2}$ & $\operatorname{Pr}(>\mathbf{F})$ \\
\hline \multicolumn{3}{|l|}{ Bray-Curtis } \\
\hline Microhabitat & 0.38595 & 0.00020 \\
\hline Treatment & 0.04911 & 0.05559 \\
\hline Microhabitat:Treatment & 0.06623 & 0.01540 \\
\hline
\end{tabular}




\section{Figure 1}

Figure 1. Boxplot of alpha diversity indexes.

Alpha diversity Observed genera, Chaol and Shannon indexes, of Bulk soil, Barke and Morex, at three different gramine concentrations. Individual dots depict individual biological replicates; no significant differences observed for gramine treatment upon Kruskal-Wallis test followed by Dunn's post-hoc test, individual p 500 values $>0.05$, Bonferroni corrected. 

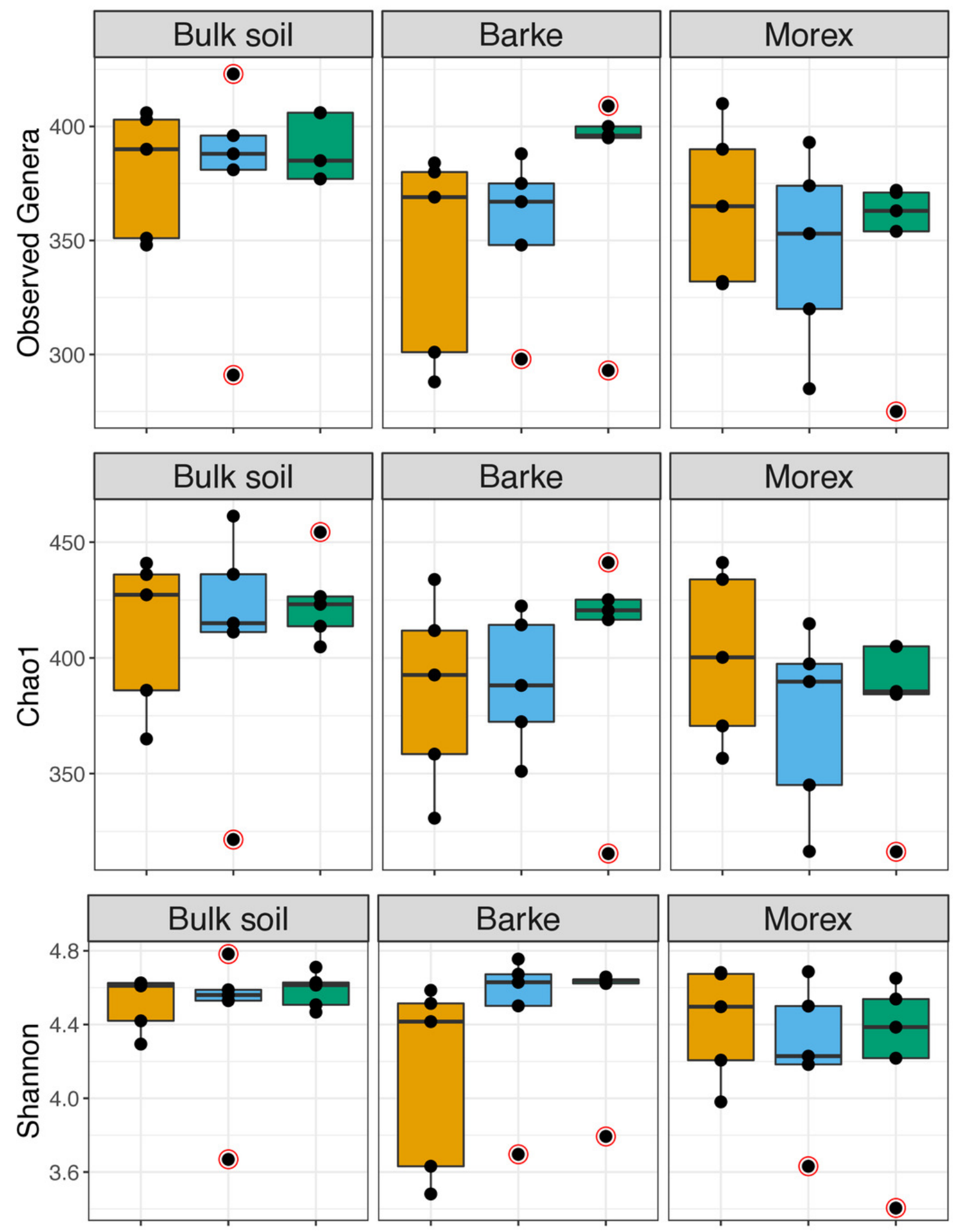

Concentration G0 G24 G 
Figure 2

Figure 2. Canonical analysis of Principal Coordinates (CAP) constructed on a Bray-Curtis dissimilarity matrix

of Bulk soil, Barke and Morex, at three different gramine concentrations Individual shapes depict individual samples, color-coded according the gramine treatment imposed on them. The ordination was constrained for genotype and gramine concentrations. 

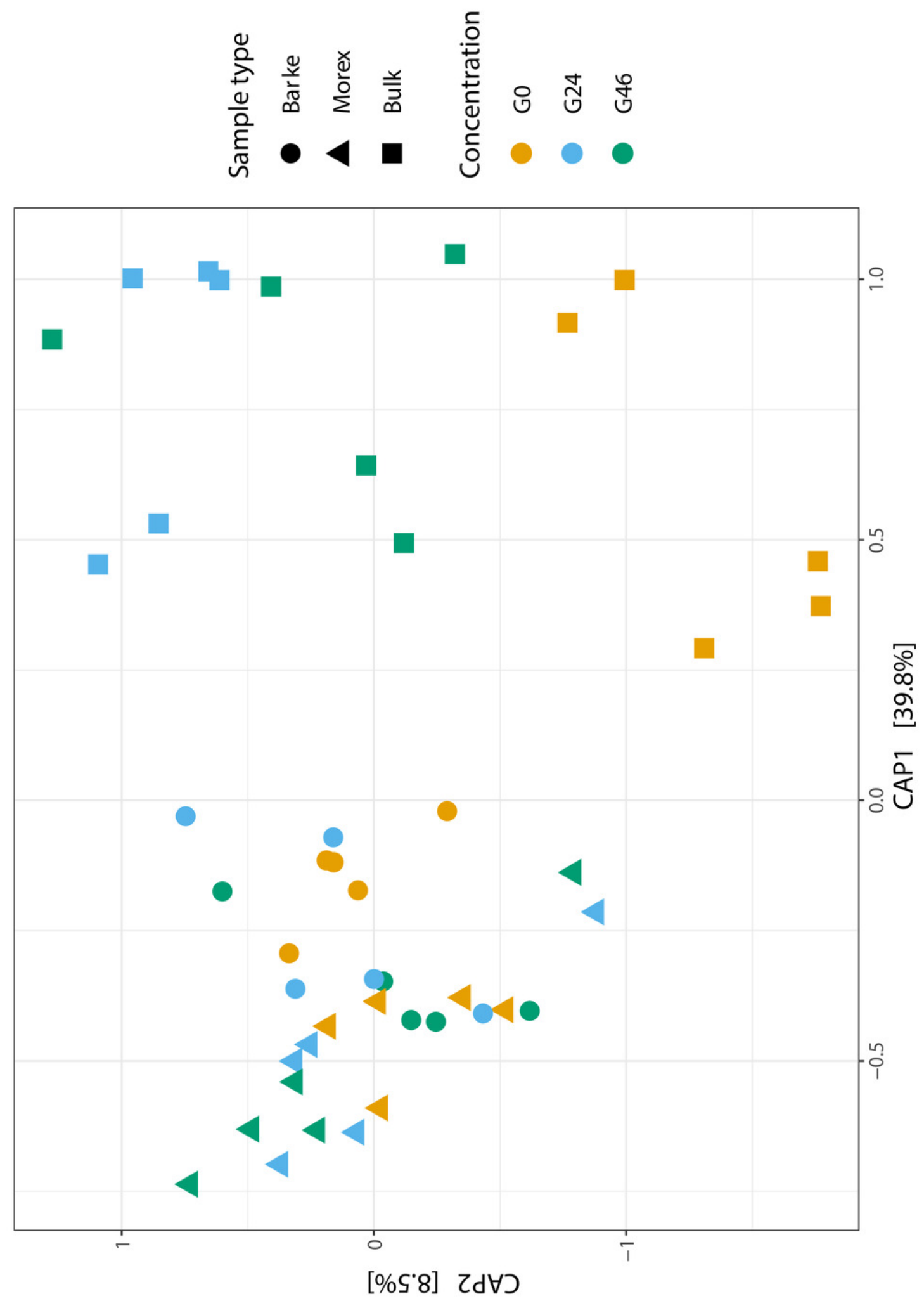


\section{Figure 3}

Figure 3. Gramine modulates bacterial abundances at the barley root-soil interface UpSetR plots of A) Genera simultaneously enriched in pair-wise comparisons retrieved from unplanted soil and Morex rhizosphere, B) and from unplanted soil and Barke rhizosphere, at the three levels of gramine tested. Vertical bars denote the number of genera enriched shared or unique for each comparison, while the horizontal bars the number of genera enriched in the indicated gramine concentration. In A and B genera differentially enriched at individual $p$ values $<0.05$, Wald Test, FDR corrected. 

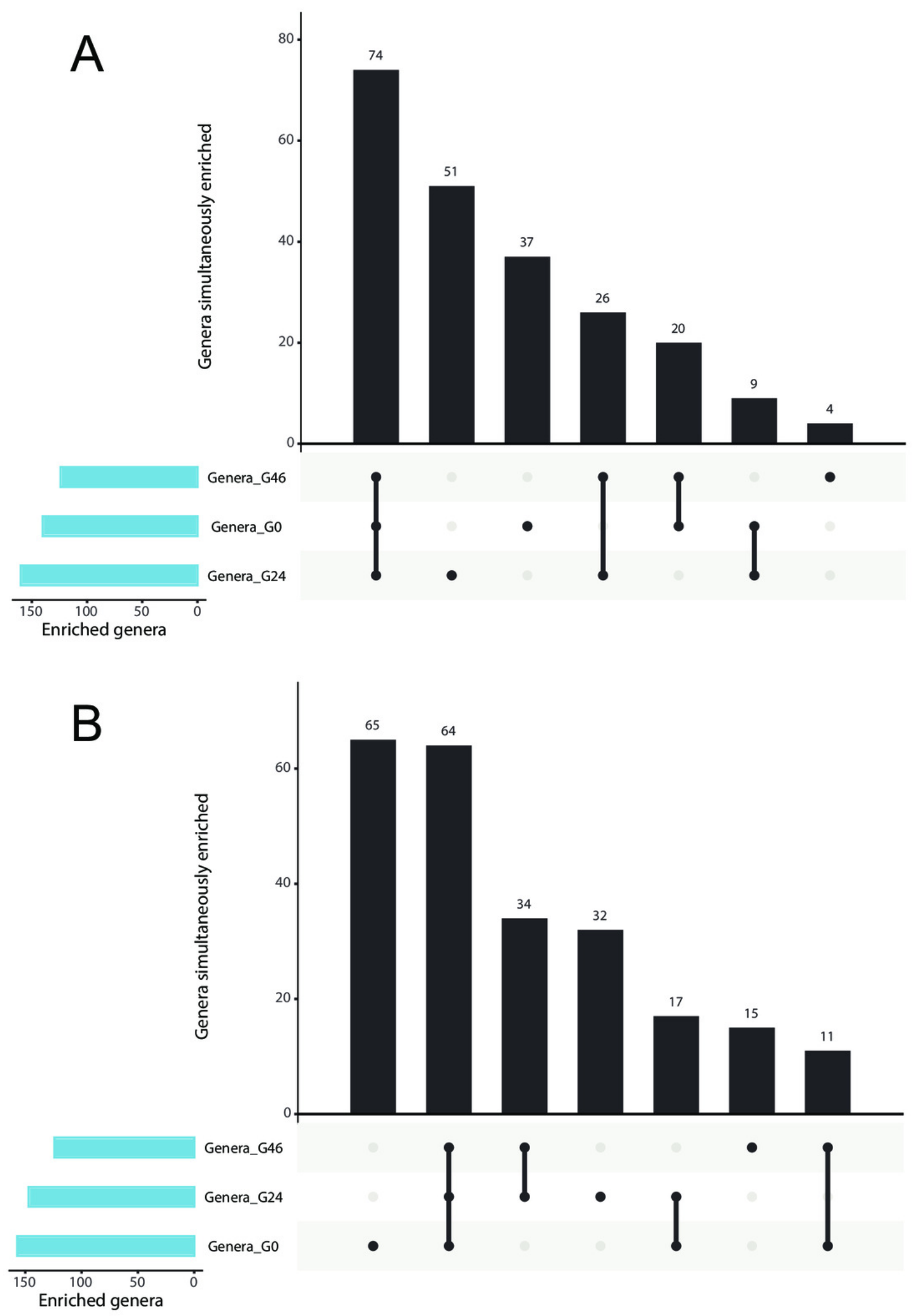

PeerJ reviewing PDF | (2021:07:64033:1:0:NEW 5 Oct 2021) 


\section{Figure 4}

Figure 4. Taxonomy and abundances of the gramine-responsive genera

Heatmap of the 18 bacteria, classified either at Order or Phylum level, significantly enriched in rhizosphere samples in a genotype- and gramine dosage- (24 and $46 \mu \mathrm{M}$, respectively) independent manner. Microbial enrichments defined at individual $p$ values $<0.05$, Wald Test, FDR corrected. 


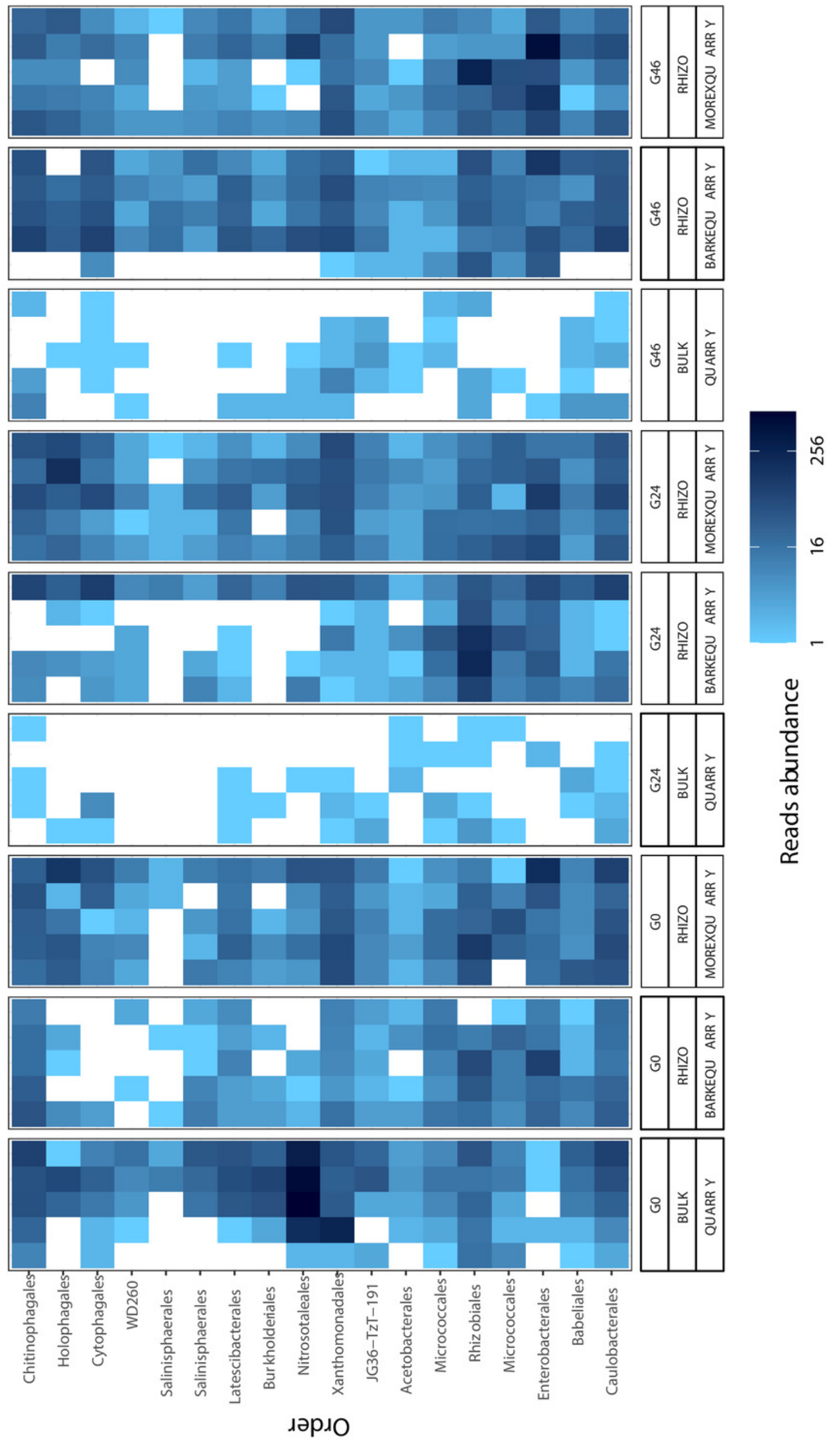




\section{Figure 5}

Figure 5. Gramine application attenuates the genotype effect on the rhizosphere microbiota

Ternary plots depicting bacteria distribution across the indicated microhabitats in sample exposed to A) no gramine, B) gramine $24 \mu \mathrm{M}$ or C) gramine $46 \mu \mathrm{M}$. In each plot, individual dots depict individual microbes whose size is proportional their sequencing abundances. Position of the dots within the plots reflects the contribution of each microhabitats to microbial abundances. Coloured dots denote genera differentially enriched between Morex and Barke (individual $p$ values $<0.05$, Wald Test, FDR corrected) color-coded according to their taxonomic affiliation at phylum level. 

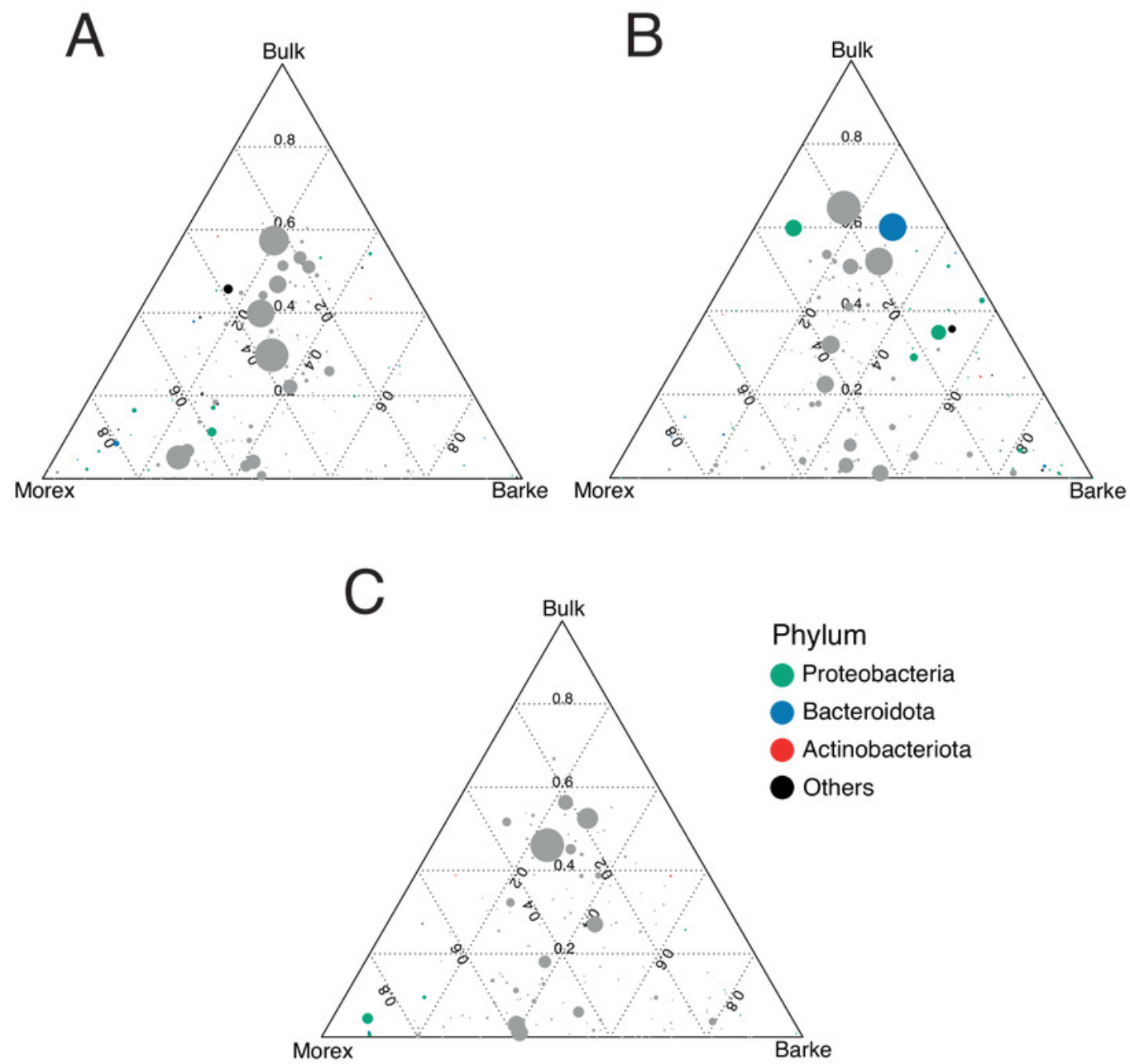